\title{
Zone Fare System Design in a Rail Transit Line
}

\author{
Yi Yang, ${ }^{1,2}$ Lianbo Deng $\mathbb{D}^{2,3}$ Qing Wang, ${ }^{2,3}$ and Wenliang Zhou ${ }^{2,3}$ \\ ${ }^{1}$ School of Transportation and Logistics, Southwest Jiaotong University, Chengdu 611756, China \\ ${ }^{2}$ School of Traffic and Transportation Engineering, Central South University, Changsha 410075, China \\ ${ }^{3}$ Rail Data Research and Application Key Laboratory of Hunan Province, Central South University, Changsha 410075, China \\ Correspondence should be addressed to Lianbo Deng; lbdeng@csu.edu.cn
}

Received 2 November 2019; Revised 12 November 2020; Accepted 16 November 2020; Published 4 December 2020

Academic Editor: Yuan Gao

Copyright ( $) 2020$ Yi Yang et al. This is an open access article distributed under the Creative Commons Attribution License, which permits unrestricted use, distribution, and reproduction in any medium, provided the original work is properly cited.

\begin{abstract}
As a widely existing form of public transit fare structures, zone fare system is traditionally designed in a separate way, which may lead to suboptimal results. This paper aims to concurrently address the zone division and fare calculation issues of the zone fare system design in a rail transit line. It is necessary to consider passengers as well as operators to find an impartial zone fare system. A fair zone fare system is one where the zone fares are as close as possible to the distance-based fares, for the fares of the distancebased fare system are highly correlated with the actual distance of trips. Thus, the fare deviations for the trips between the zone fare system and the distance-based fare system are utilized as the evaluation metric of fairness. To achieve the goal of minimizing fare deviations for all trips, we introduce three indexes: average absolute deviation, average squared deviation, and maximum mileage fare deviation. With the three indexes as the objective functions, we develop a joint optimization algorithm where a novel zone boundary adjustment scheme is proposed as the key technique. Numerical results show that the proposed algorithm can effectively provide a joint optimal scheme and the optimal number of planned zones is 5 for Changsha Metro Line 2. The proposed algorithm can provide guidance for the practical design and adjustment of the zone fare system.
\end{abstract}

\section{Introduction}

Fare is crucial to the financial situation of the rail transit. The price unit of tickets is directly determined by fare structure, which is the base and premise of the price formation. According to the relationship between the fare and the distance, fare structure generally includes the flat fare and the graduated fare.

The flat fare assigns the same fare to all trips, which is the easiest and cheapest policy to implement. However, the flat fare is extremely unfair to the short-distance passengers, who pay the same price as the long-distance passengers. Generally, the public does not accept the flat fare. The graduated fare, by contrast, can be calculated respectively based on the distance and the number of crossed zones. Thus, the graduated fare is more equitable than the flat fare. Two types of the graduated fare are therefore commonly used: distance-based fare and zone fare.
The most frequently used approach is the distance-based fare system, where the price only depends on the travelled distance. The longer the trip, the higher the fare will be. Therefore, the distance-based fare system is often considered as fair [1-3]. However, in order to calculate a specific fare, the distance for each pair of stations needs to be available for passengers and operators. This makes the system inconvenient, especially for the big city with a large number of stations $[1,2]$.

There are two types of zone fare systems. One is the zone fare system with arbitrary prices, such as the system in the north of San Francisco [1], where the fare is given arbitrarily for each pair of zones. The other is the counting zone fare system. As the most popular type of zone fare system, it has been widely accepted. Examples of such a zone fare system can be found in Oslo [4] and in the south of San Francisco [1], where the fare of a trip depends only on the crossed zones on the trip. In other words, crossing the same number 
of zones, passengers must pay the same price, even if they have different starting zones and different ending zones. Because of these unique properties, zone fare system is easy to handle and can significantly facilitate passengers' travel and operators' management.

In brief, the graduated fare is more acceptable to the public than the flat fare. In terms of the graduated fare, as a very popular system in Germany and Switzerland [5], the zone fare system is simpler than the distance-based fare system [1-3]. Over the past several decades, the distancebased fare system has been extensively studied [6-15], but the research on the design of zone fare system has been neglected. To the best of our knowledge, the literature on zone fare system is scarce.

Several studies evaluated the application effect of zone fare system from the view of economics. Jansson and Angell [4] indicated that the ticket prices of zone fare system could approximately reflect the social marginal costs of the trips, because zones vary with size and location. Andrle et al. [16] showed that zone fare system could be introduced effectively in a small transit system. After the historically complex fare system of Haifa city was simplified to a five-zone fare system, Sharaby and Shiftan [17] found that the system could reduce the travel cost of passengers and attract more passengers. By using the analytic hierarchy process, Nassi and Costa [18] found that the zone fare system was the optimal mode compared to other fare systems. Moreover, with regard to exploiting the passengers' willingness to pay, Otto and Boysen [2] showed that zone fare system is quite robust compared to the flat and distance-based fare systems.

According to these studies, we find that zone fare system is an economical and effective system in public transit. Thus, it is necessary to explore how to design the zone fare system. However, the literature on zone fare system design is rare. The problem of designing zone fare system is NP-hard $[2,19]$, which includes two subproblems: zone division and fare calculation. In terms of the implemented zone fare system in Potsdam (Germany), Borndörfer et al. [20] proposed a nonlinear fare optimization model and obtained the optimal fares. Schöbel [21] and Hamacher and Schöbel [1] provided three heuristics to obtain a fair zone design scheme in linear station networks, and they successfully redesigned the fare structure in one of Germany's transport systems by processing zone division and fare calculation individually. Koháni [22] introduced a mathematical model based on counting zones and developed two approaches to determine the quality of solution. Then, Koháni [3] made the case study for Zilina Municipality with that mathematical model. But the computation time varies from few seconds up to 2 hours for one particular parameter setting, which is not efficient enough. In order to minimize the maximum fairness deviation, Babel and Kellerer [19] proposed several heuristic algorithms and presented theoretical results for special networks. Conejero et al. [23] put forward a method of the zone fare system based on finding the longest path in a weighted root tree, and they considered all possible distributions in turn to obtain the optimal solution. Pratelli [5] developed a two-level model to deal with the problems of zone division and fare calculation separately.
In summary, the previous studies mainly focused on evaluating the application effect of the zone fare system based on the cities that have implemented this fare system. In terms of the system design, the joint optimization of zone division and fare calculation has been largely ignored in the previous studies, which may undermine the benefit of the zone fare system. In light of this, we develop a novel optimization algorithm to simultaneously solve the problems of zone division and fare calculation in designing the zone fare system. We should ensure the fairness when designing a zone fare system. Thus, the zone fares of all trips should be as close as possible to the distance-based fares, for the distancebased fare system highly relates the price to the actual distance of a trip and is mostly considered as fair [1-3]. In view of this, the fare deviations between the zone fare system and the distance-based fare system can be adopted as the objective function of the algorithm [1, 19-21]. Therefore, three objective functions are introduced to evaluate the fairness: average absolute deviation, average squared deviation, and maximum mileage fare deviation. Then, with the zone boundary adjustment as the key technique, a joint optimization algorithm is proposed based on the zone division and the fare calculation. The paper not only provides a joint optimization algorithm to design the zone fare system but also a decision-making basis for the future of this system.

The main contributions of the paper are threefold: (1) we propose an innovative algorithm to concurrently process the zone division and fare calculation for the zone fare system design; (2) we introduce three indexes to evaluate the fairness of the zone fare system scheme; (3) a novel zone boundary adjustment method is put forward as the key technique in the algorithm.

The remainder of this paper is organized as follows. Section 2 analyzes the two subproblems: zone division and fare calculation. To select the objective function, the evaluation indexes of the scheme are analyzed in Section 3. In Section 4, with zone boundary adjustment as the key technique, we present a novel heuristic algorithm; In Section 5 , a numerical example is conducted on Changsha Metro Line 2. Finally, conclusions are drawn in Section 6.

\section{Analysis}

The problem can be divided into two subproblems: zone division and fare calculation. For the rail transit lines, their operating policies are independent of each other, and so are the fare policies. Therefore, the study scope can be limited to a rail transit line. Based on a line with $n$ stations, the set of stations is denoted as $S=\left\{s_{1}, s_{2}, \ldots, s_{n}\right\}$.

2.1. Zone Division. Zones are divided along the line sequentially and fares are the same for both directions. In the zone fare system, $K$ represents the number of planned zones, and the zone is expressed as $z_{k}, 1 \leq k \leq K .\left|z_{k}\right|$ denotes the number of stations within $z_{k}$. Besides, each zone contains at least one station, namely, $\left|z_{k}\right| \geq 1$. For any station $s_{i}$, it must belong to only one zone that can be denoted as $z_{k}$. Then, $z\left(s_{i}\right)=z_{k}, 1 \leq k \leq K$, and $1 \leq i \leq n . z\left(s_{i}, s_{j}\right)$ is the number of 
zones for the trip from $s_{i}$ to $s_{j}$. Thus, the first and last stations on the line respectively belong to the zones $z_{1}$ and $z_{K}$, namely, $z\left(s_{1}\right) \in z_{1}, z\left(s_{n}\right) \in z_{K}$. To ensure there is at least one station in each zone, we set $\left|z_{k}\right| \geq 1$, for $1 \leq k \leq K$.

We define $p_{i j}$ as the number of passengers from $s_{i}$ to $s_{j}$, $1 \leq i, j \leq n, i \neq j$. As discussed above, the fare deviations between the zone fare system and the distance-based fare system are used to evaluate the fairness of zone fare system. For this reason, in the study period, the impact of the fare level on the number of passengers can be ignored. That is, $p_{i j}$ is a given constant based on the real data without considering the demand elasticity. The fare of $p_{i j}$ can be expressed as $h_{i j}=h\left(z_{a}, z_{b}\right)$, where $z\left(s_{i}\right)=z_{a}, z\left(s_{j}\right)=z_{b}$.

2.2. Fare Calculation. In zone fare system, the price of a trip is determined by the starting and ending zones of the trip. In general, for passengers with the same number of crossed zones, according to whether their fares are different, two methods of the ticket prices can be calculated as follows: the zones-differential pricing and the zones-uniform pricing. For passengers with the same zone fare, their fare levels can be determined in accordance with the mean of the maximum and minimum fares, the median price, the average fare, and other ways under the distance-based fare system. The distance-based fare is formulated as follows:

$$
f_{i j}=f_{w}^{0}+f_{w} w_{i j},
$$

where $f_{w}^{0}$ and $f_{w}$ are the fixed fare and variable fare coefficients, respectively, and $w_{i j}$ is the distance from station $i$ to station $j, 1 \leq i, j \leq n, i \neq j$.

2.2.1. The Zones-Differential Pricing. The differential pricing is determined by the starting and ending zones. The fares may be different for passengers crossing the same number of zones in the trips. For the zone fare system with arbitrary prices, utilizing the average distance-based fares, the zone fare from zone $z_{a}$ to zone $z_{b}$ can be computed by

$$
h\left(z_{a}, z_{b}\right)=\frac{\sum_{s_{i} \in z_{a}} \sum_{s_{j} \in z_{b}} f_{i j} p_{i j}}{\sum_{s_{i} \in z_{a}} \sum_{s_{j} \in z_{b}} p_{i j}} .
$$

2.2.2. The Zones-Uniform Pricing. The uniform pricing is determined only by the number of crossed zones. For the counting zone fare system, the zone fare $h(k)$ is expressed as

$$
h(k)=\frac{1}{2}\left[\max _{z\left(s_{i}\right)-z\left(s_{j}\right)=k}\left(f_{w}^{0}+f_{w} w_{i j}\right)+\min _{z\left(s_{i}\right)-z\left(s_{j}\right)=k}\left(f_{w}^{0}+f_{w} w_{i j}\right)\right] \text {, }
$$

where $z\left(s_{i}\right)-z\left(s_{j}\right)=k$ indicates the trips from station $s_{i}$ to station $s_{j}$ with $k$ crossed zones.

In general, the zones-differential pricing method is used to calculate the zone fares for a given zone division scheme, and the zone fare level is determined according to the average distance-based fare in the following sections. Moreover, the following optimization methods can be easily extended to the zone-uniform pricing methods and other pricing means.

\section{Objective Function}

As a simplified form of the distance-based fare system [1-3], the rationality of a zone fare system scheme can be reflected in the difference between the two fares. Inspired by the previous studies [1, 19-21], the evaluation indexes are shown below. $P=\sum_{s_{i}, s_{j} \in S} P_{i j}$ represents the total passenger flow. The scheme of zone fare system becomes better as the index becomes smaller and more stable.

3.1. Average Absolute Deviation ( $A A D)$. The average absolute deviation can be calculated as follows:

$$
b_{1}=\left(\frac{1}{P}\right) \sum_{s_{i}, s_{j} \in S} p_{i j}\left|h_{i j}-\left(f_{w}^{0}+f_{w} w_{i j}\right)\right| .
$$

3.2. Average Squared Deviation (ASD). In addition to the average absolute deviation, the average squared deviation can be used as another evaluation index. It is calculated as follows:

$$
b_{2}=\left(\frac{1}{P}\right) \sum_{s_{i}, s_{j} \in S} p_{i j}\left|h_{i j}-\left(f_{w}^{0}+f_{w} w_{i j}\right)\right|^{2} .
$$

3.3. Maximum Mileage Fare Deviation. Passengers who travel from $z_{a}$ and $z_{b}$ pay the same price in zone fare system, while the fares may be different for the passengers in the distance-based fare system due to their different travel distances. The maximum value of these fare deviations is denoted as the maximum mileage fare deviation $b_{\max -\min }$. For the passengers travelling from $z_{a}$ and $z_{b}$, we calculate the maximum difference between the highest distance-based fares and the lowest distance-based fares. The maximum difference can be used to compute $b_{\max -\min }$ :

$$
b_{\max -\min }=\frac{1}{2} \max _{z_{a}, z_{b} \in Z}\left[\max _{i \in z_{a}, j \in z_{b}}\left(f_{w}^{0}+f_{w} w_{i j}\right)-\min _{i \in z_{a}, j \in z_{b}}\left(f_{w}^{0}+f_{w} w_{i j}\right)\right] \text {. }
$$

We take $b_{1}, b_{2}$, and $b_{\max -\min }$ as the objective function, respectively. When one of them reaches the minimum, the optimal solution is obtained.

\section{Algorithm}

According to the characteristics of the zone fare system, we analyzed at the beginning, a joint optimization algorithm is designed to deal with the problems of zone division and fare calculation.

For a zone division scheme, zone fare $h\left(z_{a}, z_{b}\right)$ $\left(z_{a}, z_{b} \in Z\right)$ is calculated with equation (2).

When moving the zone boundary, the number of crossed zones changes. Because the affiliation's change of a boundary station between two adjacent zones will change the zone 
division scheme, the improved solutions can be achieved by adjusting the zone boundary. The most suitable objective function $b$ for a specific scenario will be selected as the goal when adjusting zone boundary.

In the left-moving operation of the boundary, passengers who have a change in the number of their crossed zones are shown in the shadow part of Figure 1(a). The boundary of $z_{k}$ and $z_{k+1}$ is $\left(s_{i}, s_{i+1}\right)$. When the boundary moves to $\left(s_{i-1}, s_{i}\right)$ along the left side of the line, station $s_{i}$ changes from $z\left(s_{i}\right)=$ $k$ to $z\left(s_{i}\right)=k+1$. Thus, the number of crossed zones will be decreased by 1 for the passengers travelling from $s_{i}$ to $\left(s_{i+1}, s_{n}\right)$, and the number of crossed zones will be increased by 1 for the passengers who travel from $\left(s_{1}, s_{i-1}\right)$ to $s_{i}$. Similarly, the right-moving operation that the boundary $\left(s_{i-1}, s_{i}\right)$ moves to $\left(s_{i}, s_{i+1}\right)$ along the right side is shown in Figure 1(b).

In the moving operation, we only consider the passengers whose number of crossed zones changes. Then, we calculate the change of the objective function value under the current level of zone fares. A boundary adjustment scheme which mostly improves the objective function can be selected as a new scheme of zone division. The fares are updated according to the new zone division scheme. An iteration is finished. In this way, the optimal scheme is finally formed. In the rail transit line, we can get the initial fare scheme when the initial zone division scheme is obtained based on the passengerkilometers (PKM) and passenger flow (PF). Thus, the initial scheme of the zone fare system is obtained.

To illustrate the algorithm more explicitly, the algorithm where the initial solution is generated based on PKM is designed as follows:

Input: station $(S)$, distance $(w)$, passenger demand $(P)$, and the number of planned zones $(K)$.

Output: zone $z\left(s_{i}\right)$ and zone fare $h\left(z_{a}, z_{b}\right)$.

(1) Calculate the PKM from the starting station to the second-to-last station on the line. That is, $T\left(s_{i}, s_{i+1}\right)=\sum_{i^{\prime}=1}^{i} \sum_{j^{\prime}=i+1}^{n} w_{i, i+1}\left(p_{i^{\prime} j^{\prime}}+p_{j^{\prime} i^{\prime}}\right)$.

The zones are divided by

$$
z\left(s_{i}\right)= \begin{cases}\frac{\sum_{i^{\prime}=1}^{i-1} T\left(s_{i^{\prime}}, s_{i^{\prime}+1}\right)}{\sum_{i^{\prime}=1}^{n-1}\left(T\left(s_{i^{\prime}}, s_{i^{\prime}+1}\right) / K\right)}+1, & 1 \leq i \leq n-1, \\ K, & i=n .\end{cases}
$$

Then, form the initial zone division scheme $Z=\left\{z\left(s_{i}\right) \mid s_{i} \in S\right\}$.

(2) Calculate and update the zone fares $h\left(z_{k}, z_{k^{\prime}}\right)=\left(\sum_{s_{i} \in z_{k}} \sum_{s_{j} \in z_{k}} f_{i j} p_{i j} / \sum_{s_{i} \in z_{k}} \sum_{s_{j} \in z_{k}} p_{i j}\right)$, $1 \leq k \leq K$.

(3) Set $k=1$, get the current scheme $Z$, and set the best scheme so far $\bar{Z}=Z$.
(4) Explore the neighborhood of current scheme by moving left and right. If $z\left(s_{i}\right)=k, z\left(s_{i+1}\right)=k+1$, let $\left(s_{i}, s_{i+1}\right)$ be the boundary point between zone $z\left(s_{i}\right)$ and zone $z\left(s_{i+1}\right)$. All boundary points move according to the following rules:

(4.1) Left-moving operation of the boundary point is $z^{-}\left(s_{i}, s_{i+1}\right)=\left\{\bar{z}\left(s_{i}\right)=z\left(s_{i}\right)+1, \bar{z}\left(s_{i+1}\right)=z\left(s_{i+1}\right)\right\}$.

In this operation, $\bar{z}\left(s_{i}\right)$ and $\bar{z}\left(s_{i+1}\right)$ represent the zones that $s_{i}$ and $s_{i+1}$ belong to, respectively. For the adjusted zone-division scheme after the left-moving operation, the number of crossed zones from $s_{i}$, and $s_{j}$, is

$$
z^{\prime}\left(s_{j^{\prime}}, s_{i^{\prime}}\right)= \begin{cases}z\left(s_{j^{\prime}}, s_{i^{\prime}}\right)+1, & i^{\prime}=i ; j^{\prime}=1, \ldots, i-1, \\ z\left(s_{j^{\prime}}, s_{i^{\prime}}\right)-1, & i^{\prime}=i ; j^{\prime}=i+1, \ldots, n, \\ z\left(s_{j^{\prime}}, s_{i^{\prime}}\right), & i^{\prime} \neq i .\end{cases}
$$

After the left-moving operation, compared with $Z$, the change of the objective function is calculated by the formula $\Delta b\left[z^{\prime}\left(s_{j}, s_{i}\right)\right]=b\left[z^{\prime}\left(s_{j}, s_{i}\right)\right]-b(Z)$.

(4.2) Right-moving operation of the boundary point is

$z^{+}\left(s_{i}, s_{i+1}\right)=\left\{\bar{z}\left(s_{i}\right)=\bar{z}\left(s_{i}\right), \bar{z}\left(s_{i+1}\right)=\bar{z}\left(s_{i+1}\right)-1\right\}$.

After the right-moving operation, the number of crossed zones from $s_{i}$, and $s_{j}$, is

$z^{\prime \prime}\left(s_{j^{\prime}}, s_{i^{\prime}}\right)= \begin{cases}z\left(s_{j^{\prime}}, s_{i^{\prime}}\right)-1, & i^{\prime}=i+1 ; j^{\prime}=1, \ldots, i, \\ z\left(s_{j^{\prime}}, s_{i^{\prime}}\right)+1, & i^{\prime}=i+1 ; j^{\prime}=i+2, \ldots, n, \\ z\left(s_{j^{\prime}}, s_{i^{\prime}}\right), & i^{\prime} \neq i+1 .\end{cases}$

After the right-moving operation, compared with $Z$, the change of the objective function is calculated by the formula $\Delta b\left[z^{\prime \prime}\left(s_{j}, s_{i}\right)\right]=$ $b\left[z^{\prime \prime}\left(s_{j}, s_{i}\right)\right]-b(Z)$.

(5) Update the solution.

(5.1) When $\quad \Delta b\left[z \prime \prime\left(s_{j}, s_{i}\right)\right] \leq \Delta b\left[z^{\prime}\left(s_{j}, s_{i}\right)\right], \quad$ if $\Delta b\left[z^{\prime \prime}\left(s_{j}, s_{i}\right)\right]<0$ and $\left|\bar{z}\left(s_{i+1}\right)\right|>1$, update $z^{+}\left(s_{i}, s_{i+1}\right)$ in $\bar{Z}$; otherwise, if $\Delta b\left[z^{\prime}\left(s_{j}, s_{i}\right)\right]<0$ and $\left|\bar{z}\left(s_{i+1}\right)\right|=1,\left|\bar{z}\left(s_{i}\right)\right|>1$, update $z^{-}\left(s_{i}, s_{i+1}\right)$ in $\bar{Z}$.

(5.2) When $\quad \Delta b\left[z^{\prime}\left(s_{j}, s_{i}\right)\right] \leq \Delta b\left[z^{\prime \prime}\left(s_{j}, s_{i}\right)\right], \quad$ if $\Delta b\left[z^{\prime}\left(s_{j}, s_{i}\right)\right]<0$ and $\left|\bar{z}\left(s_{i}\right)\right|>1$, update $z^{-}\left(s_{i}, s_{i+1}\right)$ in $\bar{Z}$; otherwise, if $\Delta b\left[z^{\prime \prime}\left(s_{j}, s_{i}\right)\right]<0$ and $\left|\bar{z}\left(s_{i}\right)\right|=1,\left|\bar{z}\left(s_{i+1}\right)\right|>1$, update $z^{+}\left(s_{i}, s_{i+1}\right)$ in $\bar{Z}$. 


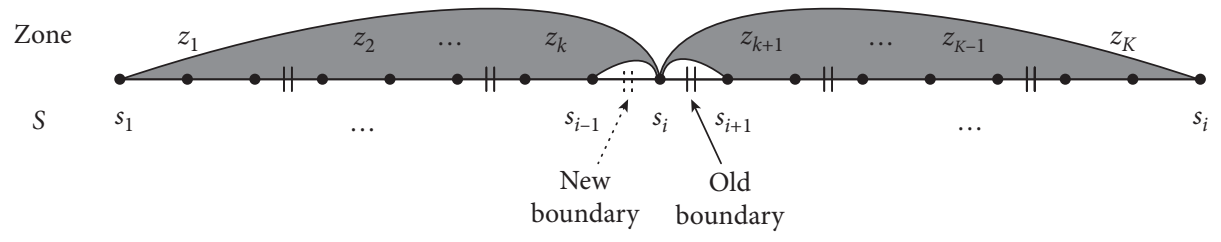

(a)

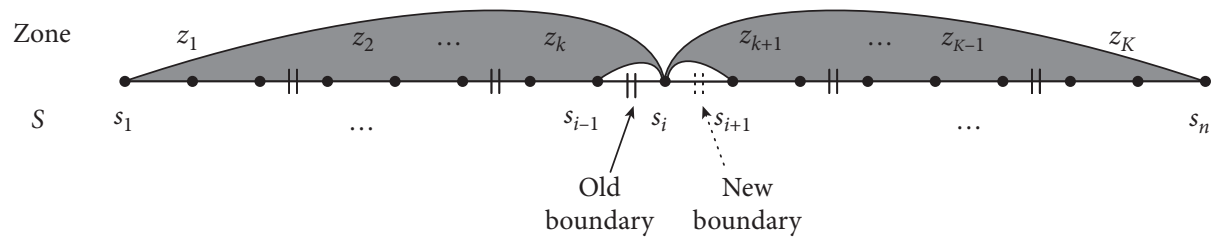

(b)

FIGURE 1: The range of the affected passengers in the moving operation. (a) The range of the affected passengers in the left-moving operation. (b) The range of the affected passengers in the right-moving operation.

(6) If $k<K$, then $k=k+1$, and go back to step (4). Otherwise, if the scheme $Z=\bar{Z}$, then the algorithm terminates and output the optimal results; if $Z \neq \bar{Z}$, then set $Z=\bar{Z}$, and go back to step (2).

The advantage of the joint optimization algorithm is that it can fill the gap of the previous studies where the zone division and the fare calculation are usually solved, respectively. Using the proposed algorithm, we can jointly address the two problems and achieve an optimal solution.

The complexity of the algorithm depends on the time required in fare calculation and the zone boundary adjustment time. The complexities of constructing the initial schemes of zone division and fare calculation are $o(n)$ and $o\left(n^{2}\right)$, respectively. There are $K-1$ boundary points in this algorithm, and the total number of the left-moving and right-moving operations is $2(K-1)$. The complexity of comparing different schemes is $n(K-1)$. The complexity of updating the new ticket prices is $o\left(n^{2}\right)$. Therefore, the sum of the algorithm complexity is $o\left(\lambda K n^{3}\right)$, where $\lambda$ represents the number of iterations when the algorithm finally converges. The algorithm efficiency can be calculated according to $\lambda$.

\section{Case Study}

5.1. Background. As shown in Figure 2, there are 19 stations in the Changsha Metro Line 2, and the length of the line is $w\left(s_{1}, s_{19}\right)=21.36 \mathrm{~km}$. The number in the gap between each pair of stations indicates the station distance in meters. In the current distance-based fare system, the fare can be computed by $f(i, j)=2+0.24 w(i, j)$. The fare unit is yuan (¥) in China.

\subsection{Scenario of 5 Planned Zones under the Objective Function} $\mathbf{b}_{2}$. To clearly illustrate the algorithm, we first study the scenario where the goal is to design 5 zones in a zone fare system with arbitrary prices. The initial solution is constructed based on the passenger-kilometers (PKM), and we take $b_{2}$ as the objective function, i.e., $b=b_{2}$. In the optimal scheme, the value of the objective function is $b_{2}=0.15$. Figure 3 shows the optimal zone division, where stations $1-4,5-9,10-12,13-15$, and 16-19 belong to zones $1,2,3,4$, and 5 , respectively. The corresponding optimal fare scheme is shown in Table 1.

The indexes' values of the optimal scheme are shown in Table 2. The fare range is $2.15-7.13$ yuan in the distancebased fare system, while it is 2.29-5.95 yuan in the zone fare system. Besides, the values of AAD and ASD are very small, which are respectively 0.31 yuan and 0.15 yuan. The indexes indicate that the zone fares are fair close to the corresponding distance-based fares for most trips. Thus, we can conclude that this scheme of the zone fare system is feasible. Moreover, figure 4 shows that the objective function can converge rapidly as the number of iterations increases. Therefore, the algorithm is proved to be convergent and efficient.

To explore the application of the proposed algorithm for different initial schemes, we construct an initial zone division scheme based on the passenger flow (PF). By taking $b_{2}$ as the objective function, it can be found that the optimal zone division scheme obtained from the initial zone division scheme under PF is the same as that under PKM. Figure 4 indicates that the algorithm efficiency under PF is similar to that under PKM, and the optimal objective function values are almost equal. Therefore, it can be concluded that the algorithm is applicable for different initial schemes.

5.3. Analysis under K Planned Zones. In order to further investigate the practical application, the number of planned zones is expanded to $K \in[3,13]$. Figure 5 shows the optimal zone division schemes obtained by using the three objective functions. Figure 5(a) depicts that the optimal schemes obtained by taking $b_{1}$ and $b_{2}$ as the objective functions are exactly the same. Besides, as the number of planned zones $K$ changes from 3 to 13 , the corresponding optimal zone division schemes under $b_{\max -\min }$ are presented in Figure 5(b). 


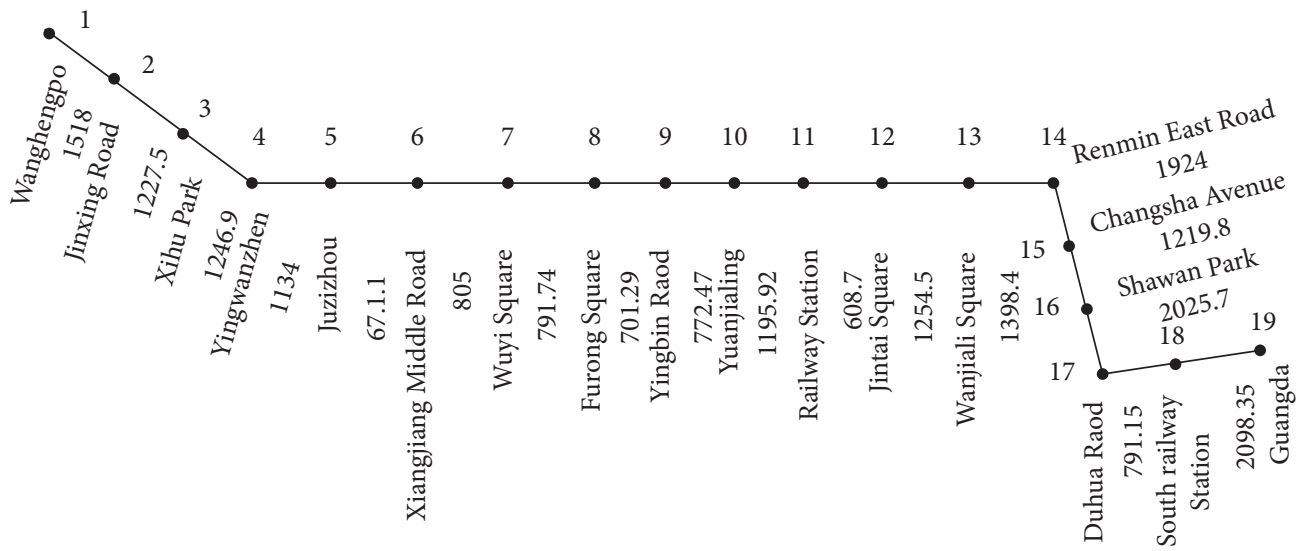

Figure 2: Changsha Metro Line 2.

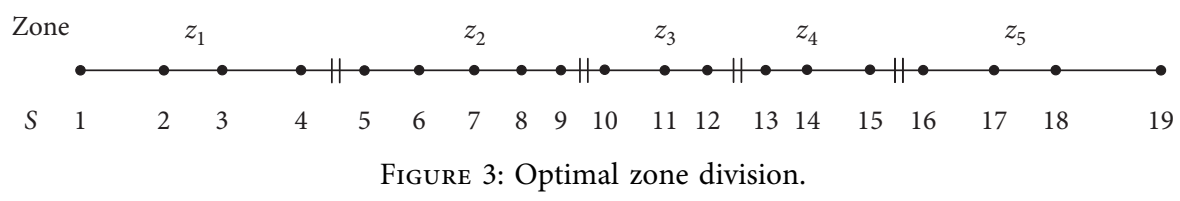

TABLE 1: Optimal zone fares.

\begin{tabular}{lccccc}
\hline Zone & 1 & 2 & 3 & 4 & 5 \\
\hline 1 & 2.57 & 3.10 & 3.81 & 4.63 & 3.47 \\
2 & 3.10 & 2.31 & 2.67 & 2.80 & 4.80 \\
3 & 3.80 & 3.48 & 2.29 & 2.53 & 4.11 \\
4 & 4.63 & 4.80 & 4.80 & 3.29 & \\
5 & 5.95 & 4.11 & 2.60 \\
\hline
\end{tabular}

TABLE 2: Evaluation of the optimal zone fare system scheme.

\begin{tabular}{lccccc}
\hline Evaluation index & Average fare & Average absolute deviation (AAD) & Average squared deviation (ASD) & Highest fare & Lowest fare \\
\hline Value & 3.52 & 0.31 & 0.15 & 5.95 & 2.29 \\
\hline
\end{tabular}

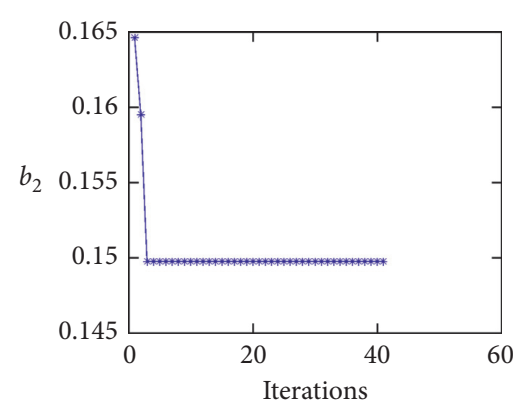

(a)

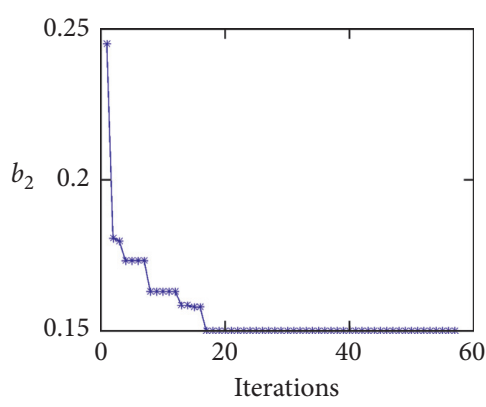

(b)

Figure 4: Convergences of the initial schemes under PKM and PF. (a) The value of $b_{2}$ under PKM. (b) The value of $b_{2}$ under PF.

Generally, Figure 5 indicates that the method is available for various numbers of planned zones.

The main results under different numbers of planned zones are analyzed as follows.
5.3.1. Range of the Fares in the Optimal Solutions. Figure 6(a) presents the highest and lowest zone fares obtained by respectively taking $b_{1}$ and $b_{2}$ as the objective functions. The highest and lowest distance-based fares are 


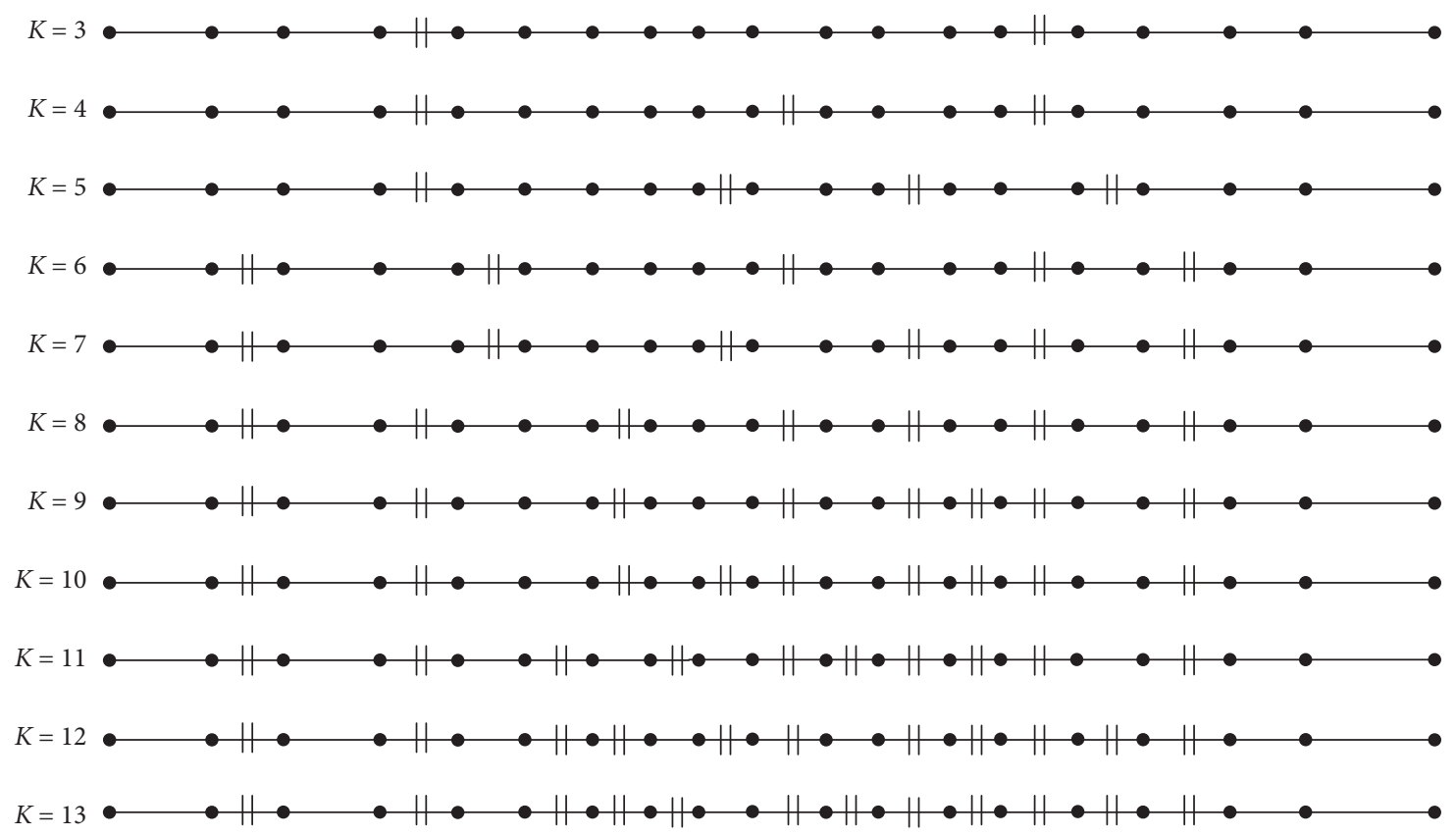

(a)
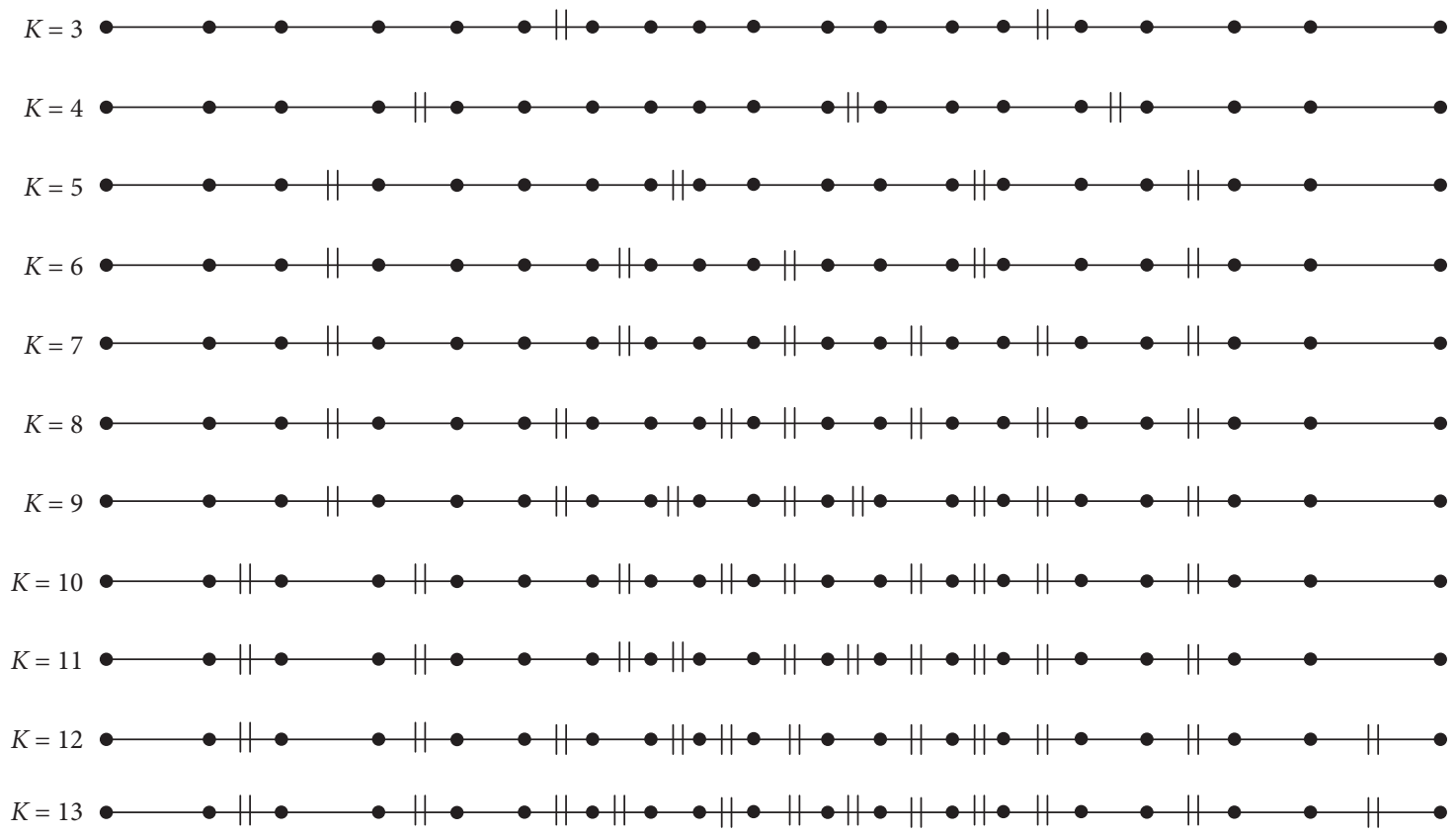

(b)

Figure 5: Optimal zone division schemes under $b_{1}, b_{2}$, and $b_{\max -\min }$ for $K \in[3,13]$. (a) Optimal zone division schemes under $b_{1}$ and $b_{2}$. (b) Optimal zone division schemes under $b_{\max -\min }$.

7.13 yuan and 2.15 yuan. Figure $6(\mathrm{a})$ indicates that the curves corresponding to $b_{1}$ and $b_{2}$ are exactly same in the zone fare system for $K \in[3,13]$, where $K=5$ is an obvious inflection point. Figure 6(b) shows the curves of the highest and lowest fares obtained by taking $b_{\max -\min }$ as the objective function, where $K=5$ is also a significant inflection point. In general, the zone fares are both gradually approaching to the distance-based fares as $K$ increases, which conforms to an expected result of the algorithm that fare deviations between the zone fares and the distance-based fares should be small.
5.3.2. Values of the Objective Functions. As shown in Figure 6(c), the three curves denote the values of $b_{1}, b_{2}$, and $b_{\max -\min }$ for $K \in[3,13]$. The trend of curve $b_{1}$ is similar to that of curve $b_{2}$, and both of them are smoother than that of $b_{\max -\min }$. All the three curves have a downward trend from fast to slow, and the inflection points of them are $K=5$.

5.3.3. Average Absolute Deviation (AAD) and Average Squared Deviation (ASD). In Figure 6(d), the blue and red curves show the values of AAD and ASD, respectively; these 

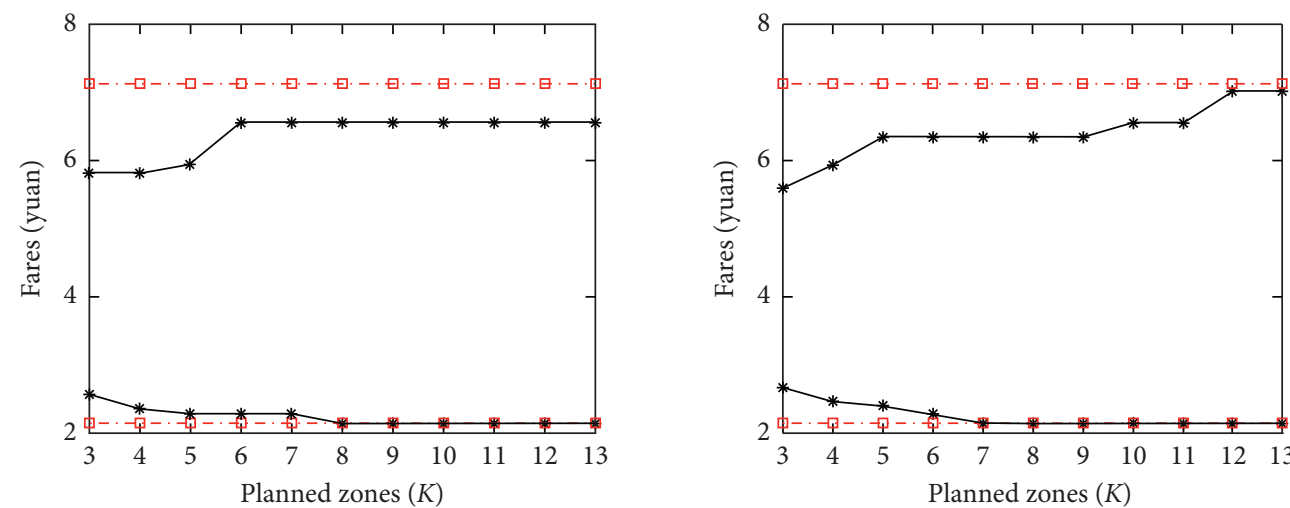

* Zone fares

$-\square-$ Distance-based fares

$\rightarrow$ Zone fares

$-\square-$ Distance-based fares

(a)

(b)
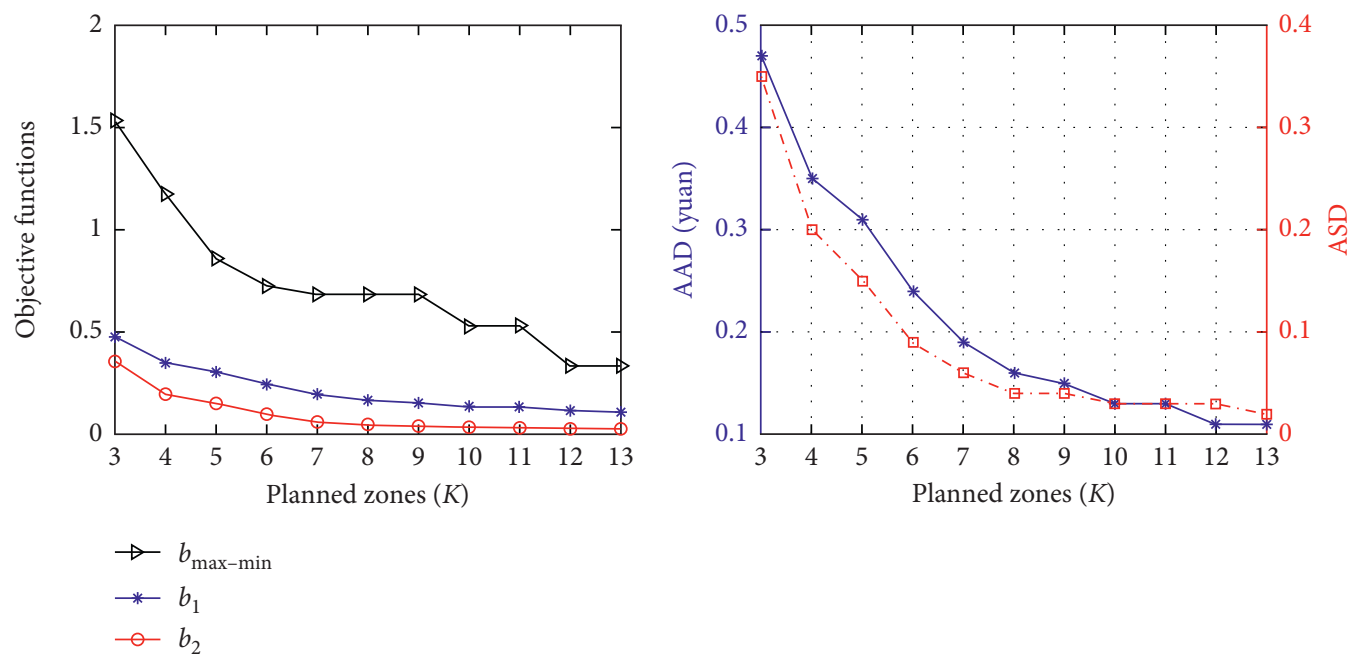

(c)

(d)

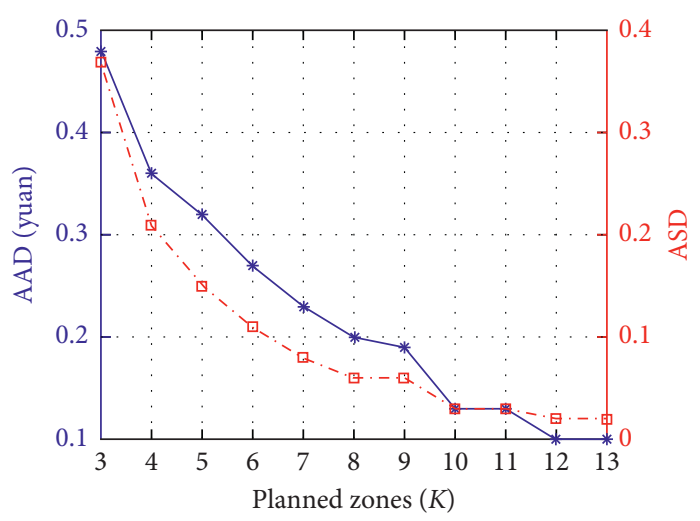

(e)

Figure 6: Analysis under objective functions $b_{1}, b_{2}$, and $b_{\max -\min }$ for $K \in[3,13]$. (a) Fare ranges under $b_{1}$ and $b_{2}$. (b) Fare range under $b_{\max -\min }$. (c) Objective functions. (d) AAD and ASD under $b_{1}$ and $b_{2}$. (e) AAD and ASD under $b_{\max -\min }$.

values are obtained by taking $b_{1}$ and $b_{2}$ as the objective functions for $K \in[3,13]$. Similarly, Figure $6(\mathrm{e})$ presents the curves of AAD and ASD when the objective function is $b_{\max -\min }$. Figures $6(\mathrm{~d})$ and $6(\mathrm{e})$ show that, as the number of planned zones increases, AAD and ASD corresponding to the three objective functions gradually decrease. Besides, the curves in Figures 6(d) and 6(e) have the downward trend from fast to slow, and the spacing between the two curves becomes obvious when $K=5$. Thus, the optimal number of planned zones is 5. Figures 6(d) and 6(e) show that both $b_{1}$ and $b_{2}$ share the same value of $\mathrm{AAD}$ when $K=5$, and the value is slightly smaller than that under $b_{\max -\min }$. Therefore, 
compared with $b_{\max -\min }, b_{1}$ and $b_{2}$ are speculated to be more suitable to be the objective function in practice.

In summary, Figures 6(a)-6(e) show that there is a significant inflection point when in this scenario $K=5$. In terms of fares concerned by operators and passengers, compared with the distance-based fares, the highest and lowest zone fares are decreased by 1.18 yuan and increased by 0.14 yuan, respectively, and the average absolute deviation is 0.31 yuan. Therefore, there will be no obvious change in the interests of passengers and operators by the transition from the current distance-based fare system to a zone fare one with 5 planned zones, and it can be speculated that this zone fare system scheme is acceptable to operators and passengers. Meanwhile, as mentioned above, zone fare system has the advantage of greatly facilitating the travel of passengers and the management of operators. Therefore, we recommend that the optimal number of planned zones is 5 for Changsha Metro Line 2 if the operator plans to implement a zone fare system, and the optimal results of zone division and fare calculation for 5 planned zones are presented in Section 5.2.

\section{Conclusions}

In the paper, we study the problems of zone fare system design in a rail transit line. Considering the interaction between zone division and fare calculation, a joint optimization algorithm is proposed. In order to test the algorithm, the paper conducts a case study on Changsha Metro Line 2 based on real data. The conclusions are drawn as follows:

(1) The optimal number of planned zones: through the theoretical and empirical research, the analysis of the solutions under the three objective functions shows that the optimal number of planned zones is 5 for Changsha Metro Line 2.

(2) Analysis of the three objective functions: the experimental results indicate that the solutions obtained by taking $b_{1}$ and $b_{2}$ as the objective functions are quite similar, and the values of AAD under $b_{1}$ and $b_{2}$ are both slightly smaller than that under $b_{\max -\min }$. In general, $b_{1}$ and $b_{2}$ are more suitable to be adopted as the objective function than $b_{\max -\min }$ in practice.

(3) The zone fare system we designed is acceptable and the algorithm is convergent and applicable. Comparing with the fares in the current fare system of the Changsha Metro Line 2, AAD and ASD indicate that there is no significant change in fares for most trips in the zone fare system. Thus, the algorithm generates an impartial zone fare system. Then, with regard to the property of simplicity as mentioned above, both operators and passengers will tend to accept the reform when the current distance-based fare system is transformed to the zone fare system. Therefore, the zone fare system we designed is acceptable. The algorithm is proved to be convergent and the algorithm complexity is $o\left(\lambda \mathrm{Kn}^{3}\right)$. Moreover, as shown in Sections 5.2 and 5.3, respectively, the algorithm proffered can not only be applied to different initial schemes under PKM and PF but also is available for various numbers of planned zones. Thus, the algorithm has a fairly good applicability for different contexts. Besides, it is easy to implement with a short computing time.

To the best of our knowledge, it is the first time that the zone division and fare calculation issues are concurrently addressed with a zone boundary adjustment as the key technique. It is worth noting that the choice of the fare system is not constant, which depends on the specific scenario of the rail transit systems and is not the focus of this study. In the future, we will explore the factors that affect the fare system and the corresponding application environment. In terms of the practical application, the study can provide a useful reference for future studies and cities that plan to implement the zone fare system.

\section{Data Availability}

The data used to support the findings of this study are available from the corresponding author upon request.

\section{Conflicts of Interest}

The authors declare that they have no conflicts of interest.

\section{Acknowledgments}

This research was supported by the National Natural Science Foundation of China (Grant nos. 71471179, U1834209, and 71871226) and the Research Fund for Fok Ying Tong Education Foundation of Hong Kong (Project no. 132017).

\section{References}

[1] H. W. Harnacher and A. Schöbel, "Design of zone tariff systems in public transportation," Operations Research, vol. 52, no. 6, pp. 897-908, 2004.

[2] B. Otto and N. Boysen, "Zone-based tariff design in public transportation networks," Networks, vol. 69, no. 4, pp. 349-366, 2017.

[3] M. Koháni, "Tariff zones design in integrated transport systems : a case study for the Zilina municipality," Mechanics, Energy, Environment, vol. 1, pp. 91-97, 2015.

[4] K. Jansson and T. Angell, "Is it possible to achieve both a simple and efficient public transport zone fare structure? Case study Oslo," Transport Policy, vol. 20, pp. 150-161, 2012.

[5] A. Pratelli, "The combined zone and fare planning problem," WIT Urban Transport X: Urban Transport and the Environment, vol. 75, no. 1, pp. 311-320, 2004.

[6] M. S. Daskin, "Review of transit service and pricing options," Journal of Advanced Transportation, vol. 17, 1983.

[7] J.-H. Ling, "Transit fare differentials: a theoretical analysis," Journal of Advanced Transportation, vol. 32, no. 3, pp. 297314, 1998.

[8] F. M. Tsai, S. I. J. Chien, and L. N. Spasovic, "Optimizing distance-based fares and headway of an intercity transportation system with elastic demand and trip length 
differentiation," Transportation Research Record, vol. 2089, no. 1, pp. 101-109, 2008.

[9] D. Yook and K. Heaslip, "Determining appropriate fare levels for distance-based fare structure," Transportation Research Record: Journal of the Transportation Research Board, vol. 2415, no. 1, pp. 127-135, 2014.

[10] Z.-C. Li, W. H. K. Lam, S. C. Wong, and A. Sumalee, "Design of a rail transit line for profit maximization in a linear transportation corridor," Transportation Research Part E: Logistics and Transportation Review, vol. 48, no. 1, pp. 50-70, 2012.

[11] C. Tang, A. Ceder, and Y.-E. Ge, "Integrated optimization of bus line fare and operational strategies using elastic demand," Journal of Advanced Transportation, vol. 2017, Article ID 7058789, 15 pages, 2017.

[12] S. Farber, K. Bartholomew, X. Li, A. Páez, and K. M. Nurul Habib, "Assessing social equity in distance based transit fares using a model of travel behavior," Transportation Research Part A: Policy and Practice, vol. 67, pp. 291-303, 2014.

[13] W. H. K. Lam and J. Zhou, "Optimal fare structure for transit networks with elastic demand," Transportation Research Record: Journal of the Transportation Research Board, vol. 1733, no. 1, pp. 8-14, 2000.

[14] D. Yang, E. Kastrouni, and L. Zhang, "Equitable and progressive distance-based user charges design and evaluation of income-based mileage fees in Maryland," Transport Policy, vol. 47, pp. 169-177, 2016.

[15] F. Zhu and S. V. Ukkusuri, "A reinforcement learning approach for distance-based dynamic tolling in the stochastic network environment," Journal of Advanced Transportation, vol. 49, no. 2, pp. 247-266, 2015.

[16] S. Andrle, J. Kraus, and F. Spielberg, "Lessons from the broome county distance-based fare Demonstration : effects of zone fares and off-peak discounts on ridership, revenue, pass sales, and public opinion," Transportation Research Record, vol. 1297, pp. 50-56, 1991.

[17] N. Sharaby and Y. Shiftan, "The impact of fare integration on travel behavior and transit ridership," Transport Policy, vol. 21, pp. 63-70, 2012.

[18] C. D. Nassi and F. C. d. C. d. Costa, "Use of the analytic hierarchy process to evaluate transit fare system," Research in Transportation Economics, vol. 36, no. 1, pp. 50-62, 2012.

[19] L. Babel and H. Kellerer, "Design of tariff zones in public transportation networks: theoretical results and heuristics," Mathematical Methods of Operations Research (ZOR), vol. 58, no. 3, pp. 359-374, 2003.

[20] R. Borndörfer, M. Karbstein, and M. E. Pfetsch, "Models for fare planning in public transport," Discrete Applied Mathematics, vol. 160, no. 18, pp. 2591-2605, 2012.

[21] A. Schöbel, "Fair zone design in public transportation networks," Operations Research Proceedings, pp. 191-196, Springer, Berlin, Germany, 1995.

[22] M. Koháni, "Exact approach to the tariff zones design problem in public transport," in Proceedings of 30th International Conference Mathematical Methods in Economics, pp. 426-431, Karvina, Czech Republic, September 2012.

[23] J. A. Conejero, C. Jordán, and E. Sanabria-Codesal, “An iterative algorithm for the management of an electric car-rental service," Journal of Applied Mathematics, vol. 2014, Article ID 483734, 11 pages, 2014. 